\title{
Percutaneous cement augmentation for the treatment of depression fractures of the tibial plateau
}

\author{
D. S. Evangelopoulos $\cdot$ S. Heitkemper $\cdot$ S. Eggli $\cdot$ \\ U. Haupt · A. K. Exadaktylos · L. M. Benneker
}

Received: 9 July 2009/ Accepted: 13 November 2009/Published online: 15 December 2009

(C) Springer-Verlag 2009

\begin{abstract}
The management of insufficiency fractures of the tibial plateau in osteoporotic patients can be very challenging, since it is difficult to achieve a stable fixation, an essential condition for the patients' early mobilization. We present a minimally invasive technique for the treatment of proximal tibial plateau fractures, "tibiaplasty", using percutaneous polymethylmethacrylate augmentation. Five osteoporotic patients ( 7 fractures) with a non-traumatic insufficiency tibial plateau fracture were treated with this technique at the authors' institution from 2006 to 2008. The patients' median age was 79 (range 62-88) years. The intervention was performed percutaneously under general or spinal anesthesia; after the intervention, immediate full weight bearing was allowed. The technique was feasible in all patients and no complications related to the intervention were observed. All patients reported a relevant reduction in pain, were able to mobilize with full weight bearing and would undergo the operation again. No secondary loss of reduction or progression of arthrosis was observed in radiological controls; no revision surgery was required. Our initial results indicate that tibiaplasty is a good treatment option for the management of insufficiency in tibial plateau fractures in osteoporotic patients. The technique is minimally invasive, safe and allows immediate mobilization without restrictions. In our group of patients, we found excellent early to mid-term results.
\end{abstract}

D. S. Evangelopoulos $\cdot$ S. Heitkemper $\cdot$ S. Eggli $\cdot$ U. Haupt

L. M. Benneker $(\bowtie)$

Department of Orthopedic Surgery, Inselspital,

University of Bern, 3010 Bern, Switzerland

e-mail: lorin.benneker@insel.ch

A. K. Exadaktylos

Department of Emergency Medicine, Inselspital,

University of Bern, Bern, Switzerland
Keywords Depression-type fractures of tibial plateau . Percutaneous cement augmentation .

Polymethylmethacrylate (PMMA) · Tibiaplasty

\section{Introduction}

In young patients, fractures of the tibial plateau are serious, high energy, intra-articular injuries. In osteoporotic patients, on the other hand, these lesions are low energy fractures developing after normal loading. Since the subchondral bone is often sclerotic, articular involvement in insufficiency fractures of the tibial plateau is rare, as the region of lowest resistance is the metaphyseal area. Nontraumatic knee pain is very common in such patients and therefore insufficiency fractures are often not diagnosed. Radiographic imaging of such lesions includes anteroposterior and lateral views. A bone scan may also prove to be very useful in the diagnosis of insufficiency fractures in older patients $[6,15]$. In case of doubt about fracture classification and/or a trauma mechanism, an MRI or a CT scan of the knee can rule out involvement of the articular surface.

Treatment of osteoporotic tibial plateau fractures is well known to be linked with problems related to internal fixation stability due to diminished bone strength [4]. Casting, simple traction by ligamentotaxis, or external and internal fixation are some of the proposed treatment options. Nevertheless, all these techniques are linked with serious complications [23]. Vertebroplasty or kyphoplasty have been established as minimally invasive techniques for the treatment of vertebral osteoporotic fractures, with excellent results, leading to immediate and lasting pain relief in the vast majority of patients [10]. Adaptation of these 
Fig. 1 a Insertion of 2-mm $\mathrm{K}$-wires, $\mathbf{b}$ replacement by side-opening $8 \mathrm{G}$ filling cannula, c lateral view after first injection of PMMA, $\mathbf{d}$ final image, cementation is limited by extravasation into to the medullary canal

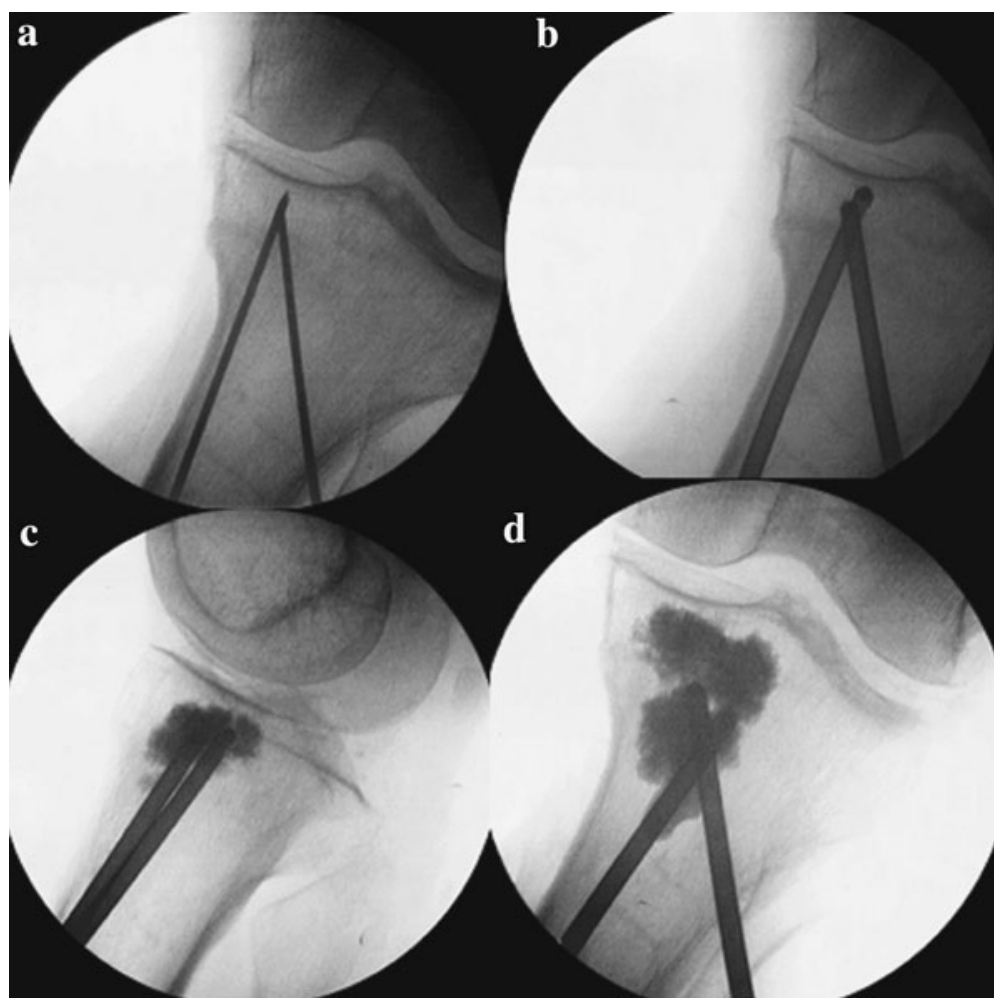

techniques for other regions, for example the proximal tibia, has not yet been reported.

The purpose of the present study was to investigate whether a "minimally invasive method" could offer appropriate stabilization and reduction for the treatment of osteoporotic fractures without endangering the soft tissue elements.

\section{Materials and methods}

Between 2006 and 2008, 5 patients with 7 non-traumatic fractures of the tibial plateau were treated at our unit with the minimally invasive technique presented here. The study group consisted of 1 male and 4 female patients, with a median age of 79 (range 62-88) years. The median followup was 3 (range 1-4) years. All patients had an episode of at least 4 weeks of persistent knee pain before presentation. Patients were not suited for conservative treatment for different reasons: 2 patients presented with bilateral insufficiency fractures, 2 patients had severe back problems that made mobilization with crutches impossible, and the fifth patient had an insufficiency fracture of the talar neck of the contralateral extremity. For all patients, anteroposterior, lateral digitalized radiographs and knee CT or MRIscans were performed. Comminutuion of the articular surface was an exclusion criterion. Varus, respectively valgus, deformity of the articular surface was measured electronically pre-, 2- and 12-month post-operatively at an accuracy of $0.1^{\circ}$ as provided by the system (PACS, Philips Electronics, Amsterdam, NL). All patients signed an informed consent form and were aware that we might have to change to an open procedure in the event of technical difficulties.

The operations were performed under general (3/5) or spinal anesthesia $(2 / 5)$ by an orthopedic surgeon with experience in cement augmentation of the spine. Patients were in the supine position on a radiolucent operating table. After disinfection and sterile draping, a single shot dose of prophylactic antibiotics (Zinacef $1.5 \mathrm{~g}$, intravenous) was administered. After a 3- to 4-mm stab incision under radiological control (Siremobil ${ }^{\circledR}$, Siemens Medical Solutions, Zürich, Switzerland), two 2-mm K-wires were advanced from a anterolateral and a anteromedial entry point approximately 5-7 cm distal of the joint line toward the tibial plateau, taking every care not to penetrate the subchondral bone. The K-wire from the anteromedial entry point was aimed toward the dorsolateral part of the medial plateau or toward the anteromedial part for the second wire (Fig. 1a). In the single patient with a lateral depression fracture, the procedure was performed in the same manner, but as the mirror image. After biplanar confirmation of correct placement, the guidewires were replaced by 8 Gauge filling cannulas designed for 
Fig. 2 Anteroposterior (a) and lateral (b) radiograph 6 months after tibiaplasty. The extravasation into the medullary canal stayed in place over time
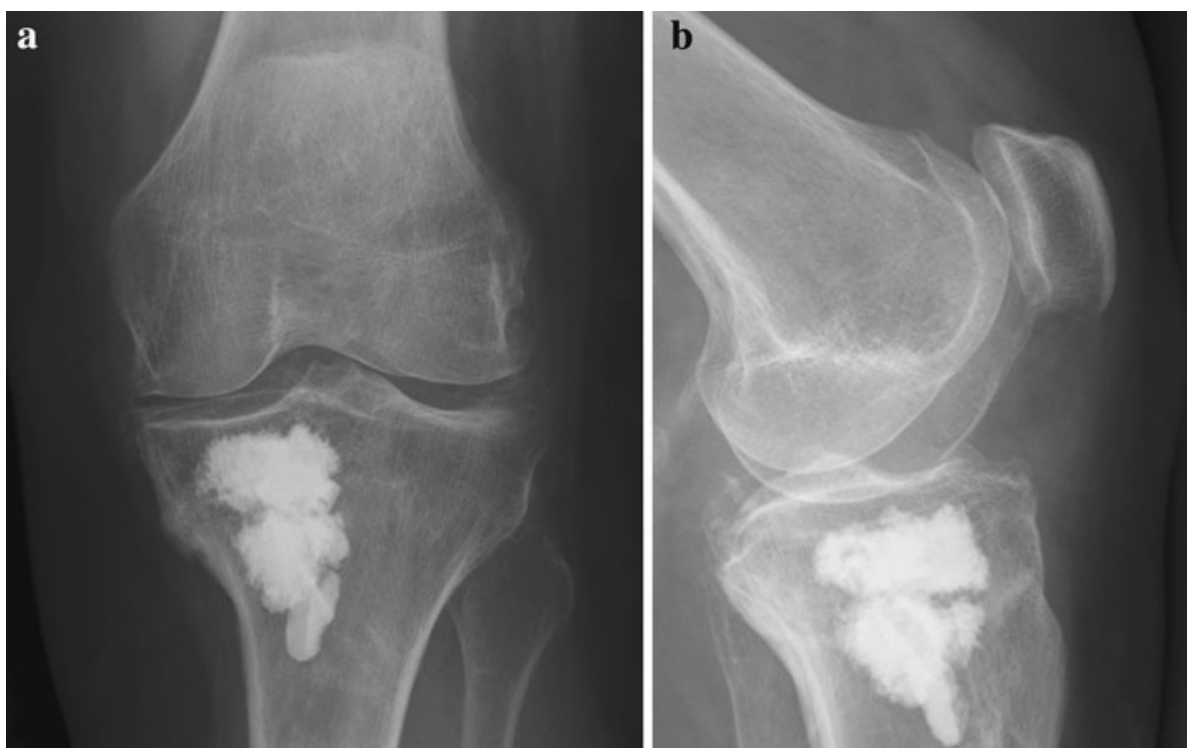

vertebroplasty. We selected side-opening cannulas (Vertecem Kit, Synthes GmbH, Switzerland), as these have closed, rounded distal ends and can be better controlled for the flow of cement through the small side-opening [11]. Under valgus stress and with gentle hammer blows on the filling cannula, partial reduction of the impacted medial plateau was attempted. Greatest care is necessary to ensure that subchondral bone is not penetrated (Fig. 1b). The cannula was then retracted about 3-5 mm and high viscous polymethylmethacrylate cement (Vertecem, Synthes Inc., Switzerland) was injected under constant fluoroscopic control. At first, only a minimal amount of polymethylmethacrylate (about $0.5 \mathrm{cc}$ for each needle) was injected and allowed to cure at body temperature for $30 \mathrm{~s}$, to minimize the risk of possible extravasations. To achieve controlled local cement distribution, both cannulas were slowly rotated $360^{\circ}$ during injection of approximately another 1-2 cc of polymethylmethacrylate each, and the needles were further retracted until the openings were at the height of the fracture. At this point, another 2-4 cc cement ( $2 \mathrm{cc}$ per needle), was injected with $360^{\circ}$ rotation. Finally, the procedure was repeated $1 \mathrm{~cm}$ distal of the fracture (Fig. 1c, d). We aimed to inject a total of $10-14 \mathrm{cc}$ of cement. In some patients, this was not possible, due to the presence of cement too close to the joint line or due to extravasations into the tibial medullar canal. After complete curing of the polymethylmethacrylate, the needles were retrieved and the incision was closed by a single stitch.

Post-operative care consisted of immediate full weight bearing, initially with the use of crutches. No limitations were applied to the range of motion and patients were allowed to leave hospital the day after the intervention.

\section{Results}

The technique was feasible in all patients and no intraoperative complications were observed. The median operation time was 30 (range 30-45) min. There were no infections or wound-healing problems in this series; one patient developed palpable ossification at the anteromedial entry point that was not symptomatic. All patients could be mobilized on the first post-operative day with the help of crutches. The range of motion was the same as of that on the pre-operative day and was symmetric to the contralateral side (where applicable). The median hospital stay was 2 (range 1-3) days (excluding one patient who underwent a major spinal intervention during the same hospitalization of 12 days). All patients reported a significant decrease in pain immediately after the operation were satisfied or very satisfied with the result after 2 months and would undergo the surgery again.

In the follow-up over a minimal 12 month period, no secondary loss of reduction or progression of the deformity was seen. Moreover, no signs of accelerated arthrosis (osteophytes, joint space narrowing, sclerosis) were observed over a period of 12 months. Cement extravasation was detected in three patients: in one patient into the soft tissues over the medial cortex and in the other two into the medullary canal (Fig. 2a, b). None of these extravasates was symptomatic. We were able to inject a mean total of $9.9 \mathrm{cc}( \pm 2.1)$ of cement.

\section{Discussion}

The most important finding of the present study was that tibiaplasty achieved acceptable and stable fracture 
reduction in the treatment of depression-type fractures of the tibial plateau, allowing immediate post-operative mobilization and full weight bearing without secondary loss of reduction. There were no serious complications.

Conservative treatment for tibia plateau fractures has been described in the literature, employing ligamentotaxis $[16,17]$. The use of a cast offers immediate ambulation and a shorter hospital stay but is not capable of providing either the range of motion or the necessary stability to prevent deformity, axial collapse and post-immobilization stiffness $[7,8]$. Furthermore, older patients, such as those in this study, are often incapable of performing partial weight bearing due to their comorbidities. Internal fixation of such fractures may offer several advantages [19], but, even with minimally invasive techniques, the skin or soft tissue necrosis caused by surgical manipulations on the soft tissue envelope-already sensitive and swollen-together with the possibility of infection may compromise the final result [18]. Additionally, the poor bone quality in osteoporotic patients necessitates the use of a bone cement to enhance fixation stability while performing osteosynthesis [2, 1214, 21, 22].

In this series, polymethylmethacrylate (PMMA) was used as it provides greater and more immediate mechanical strength than calcium phosphate cement and that it allows immediate and full weight bearing, which is mandatory for the fast mobilization of this patient group. As possible heat damage to the adjacent articular cartilage from the exothermic reaction of the curing PMMA was a concern, a safety margin of a few millimeters to the joint line was always maintained. Fortunately, there is a very steep temperature gradient, so that no heat damage to the articular cartilage would be expected even only a few millimeters beyond the cement and additionally the circulating synovial fluid is leading off the generated heat $[1,3,5,9,20]$. Of course, this technique is not recommended for young patients with high demands on their cartilage.

One limitation of this study could be considered to be the number of patients included in our study group. This limited number does not permit proper statistical analysis, so that no reliable conclusions can be drawn. A second limitation may be the lack of a direct comparison control group with early open reduction and internal fixation. This report may serve as the basis for a prospective, randomized clinical trial, although one must consider that, at least in our institution, these injuries are rarely presented and that partial weight bearing when possible is a valid alternative to surgery. In future, however, bearing the increasing aging population with osteoporosis in mind, an increase in such injuries is imaginable and a minimally invasive and relatively cheap method to maintain independence and mobility of these patients can prove to be beneficial for health care systems.

\section{Conclusion}

Closed reduction and PMMA augmentation as a definitive treatment for insufficiency fractures of the tibial plateau proved to be beneficial in selected old osteoporotic patients. The technique is feasible, shows a low complication rate and could even be performed under local anesthesia. It provided the possibility of proper osseous stabilization, without compromising the soft tissue envelope or the early mobilization of the patients.

\section{References}

1. Aebli N, Goss BD, Thorpe P, Williams R, Krebs J (2006) In vivo temperature profile of intervertebral discs and vertebral endplates during vertebroplasty - an experimental study in sheep. Spine 3:1674-1678

2. Bajammal SS, Michael Zlowodzki M, Lelwica A, Tornetta P III, Einhorn TA, Buckley R, Leighton R, Russell TA, Larsson S, Bhandari M (2008) The use of calcium phosphate bone cement in fracture treatment. A meta-analysis of randomized trials. J Bone Joint Surg Am 90:1186-1196

3. Belkoff SM, Molloy S (2003) Temperature measurement during polymerization of polymethylmethacrylate cement used for vertebroplasty. Spine 28:1555-1559

4. Biyani A, Reddy N, Chaudhury J, Simison AJ, Klenerman L (1995) The results of surgical management of displaced Tibial plateau fractures in the elderly. Injury 26:291-297

5. Boner V, Kuhn P, Mendel T, Gisep A (2009) Temperature evaluation during PMMA screw augmentation in osteoporotic bone - an in vitro study about the risk of thermal necrosis in human femoral heads. J Biomed Mater Res B Appl Biomat 90:842-848

6. Cabitza P, Tamimi H (2000) Occult fractures of tibial plateau detected employing magnetic resonance imaging. Arch Orthop Trauma Surg 120:355-357

7. DeCoster TA, Nepola JV, El-Khoury GY (1988) Cast brace treatment of proximal tibial fractures: a ten-year follow-up study. Clin Orthop Relat Res 231:196-204

8. Delamarter R, Hohl M (1989) The cast brace, tibial plateau fractures. Clin Orthop Relat Res 242:26-31

9. Deramond H, Wright NT, Belkoff SM (1999) Temperature elevation caused by bone cement polymerization during vertebroplasty. Bone 25:17S-21S

10. Heini PF (2005) The current treatment-a survey of osteoporotic fracture treatment. Osteoporotic spine fractures: the spine surgeon's perspective. Osteoporos Int 16:S85-S92

11. Heini PF, Allred CD (2002) The use of a side-opening injection cannula in vertebroplasty-a technical note. Spine 27:105-109

12. Horstmann WG, Verheyen CC, Leemans R (2003) An injectable calcium phosphate cement as a bone-graft substitute in the treatment of displaced lateral tibial plateau fractures. Injury 34:141-144

13. Keating JF, Hajducka CL, Harper J (2003) Minimal internal fixation and calcium phosphate cement in the treatment of fractures of the tibial plateau-a pilot study. J Bone Joint Surg Br 85:68-73

14. Lobenhoffer P, Gerich T, Witte F, Tscherne H (2002) Use of an injectable calcium phosphate bone cement in the treatment of tibial plateau fractures: a prospective study of twenty-six cases 
with twenty-month mean follow-up. J Orthop Trauma 16:143149

15. Prasad N, Murray JM, Kumar D, Davies SG (2006) Insufficiency fracture of the tibial plateau: an often missed diagnosis. Acta Orthop Belg 72:587-591

16. Salter RB, Ogilvie-Harris DJ (1979) Healing of intra-articular fractures with continuous passive motion. In: Cooper RR (ed) AAOS instructional course lectures, vol 28. Mosby, St Louis, pp 102-117

17. Salter RB, Simonds DF, Malcolm BW, Rumble EJ, MacMichael D, Clements ND (1980) The biological effect of continuous passive motion on the healing of full-thickness defects in articular cartilage: an experimental investigation in the rabbit. J Bone Joint Surg Am 62:1232-1251

18. Stevens DG, Beharry R, McKee MD, Waddell PJ, Schemitsch EH (2001) The long-term functional outcome of operatively treated Tibial plateau fractures. J Orthop Trauma 15:312-320
19. Tscherne H, Obenhofferm P (1993) Tibial plateau fractures. Management and expected results. Clin Orthop Relat Res 292:87-100

20. Verlaan JJ, Oner FC, Verbout AJ, Dhert WJ (2003) Temperature elevation after vertebroplasty with polymethyl-methacrylate in the goat spine. J Biomed Mater Res B Appl Biomater 67:581-585

21. Welch RD, Zhang H, Bronson DG (2003) Experimental tibial plateau fractures augmented with calcium phosphate cement or autologous bone graft. J Bone Joint Surg Am 85:222-231

22. Yetkinler DN, McClellan RT, Reindel ES, Carter D, Poser RD (2001) Biomechanical comparison of conventional open reduction and internal fixation versus calcium phosphate cement fixation of a central depressed tibial plateau fracture. J Orthop Trauma 15:197-206

23. Young MJ, Barrack RL (1994) Complications of internal fixation of tibial plateau fractures. Orthop Rev 23:149-154 